\title{
Estimating Electric Field and SAR in Tissue in the Proximity of RF Coils
}

\author{
Rosti Lemdiasov, Arun Venkatasubramanian, and Ranga Jegadeesan
}

\section{Introduction}

Inductive systems for delivering power and performing communications have become ubiquitous in medical implants [1-3]. In modern literature [4-9], there are numerous design approaches for building robust induction-based wireless links, and some of them include constraints based on exposure regulations as part of their design methodology. We find that there is a huge reliance on full-wave simulation tools such as Ansys HFSS, Remcom XFdtd, and Zurich Med Tech SIM4LIFE for exposure assessments. However, in some cases, this may be the only means to get some insights on the electromagnetic field distribution in tissues, a result of nonhomogeneous dispersive behavior of human tissues and its associated irregular stratified geometry. However, we lose the ability to get an intuitive understanding of the electromagnetic phenomena in the human body from the point of view of the basic electromagnetic principles, such as those outlined in [10, 11]. Additionally, working with full-wave simulation tools is very involved, and it requires familiarity with the tool and has a sizeable setup time and computational effort. In this paper, we present our earnest attempt to simplify exposure assessment so that the effort needed can be significantly reduced while not compromising on the degree of accuracy of results. We approach this problem by trying to estimate electric field in human body due to a nearby inductive system that has an RF coil adjacent to the human body. The

R. Lemdiasov $(\bowtie)$

Cambridge Consultants, Boston, MA, USA

e-mail: rosti.lemdiasov@cambridgeconsultants.com

A. Venkatasubramanian · R. Jegadeesan

Cambridge Consultants, Singapore, Singapore

e-mail: arun.venkat@cambridgeconsultants.com; ranga.jegadeesan@ cambridgeconsultants. com 
specific absorption rate (SAR) and induced currents can then be computed from first principles using well-established tissue properties [12] and compared with regulatory limits.

\section{SAR in the Tissue}

The magnetic field generated by the inductive systems used in medical implants induces currents in the tissues that are exposed to it. These currents generate heat and can cause tissue damage if left unchecked. To address this issue and regulate the use of induction technology so that safe operating conditions can be ensured in medical devices, exposure restrictions are enforced by the Food and Drug Administration (FDA) regulations in the United States and CE regulations in the EU. Both regulatory bodies use SAR metric as the key yardstick to evaluate field exposure. To cite the SAR regulatory limits for general exposure, the FDA has coordinated with the Federal Communications Commission (FCC) to set a limit of $1.6 \mathrm{~W} / \mathrm{kg}$ averaged over a volume containing a mass of $1 \mathrm{~g}$ of tissue for the torso and head [13]. The corresponding limit in the EU is $2 \mathrm{~W} / \mathrm{kg}$ averaged over a volume containing $10 \mathrm{~g}$ of tissue [14].

In a typical inductive charging system, the receive coil is part of the implant which is located at a certain depth inside the tissue (Fig. 1). The implant charging rate depends on the distance between the transmit coil and receive coil. At low frequencies (when skin depth in tissue is large compared to tissue size), the effect of tissue on the generated magnetic field is negligible. Hence, for purposes of estimating charging rate, tissue needs not be considered, and the system can be considered as though present in air. This also implies that the losses in tissue due to the magnetic
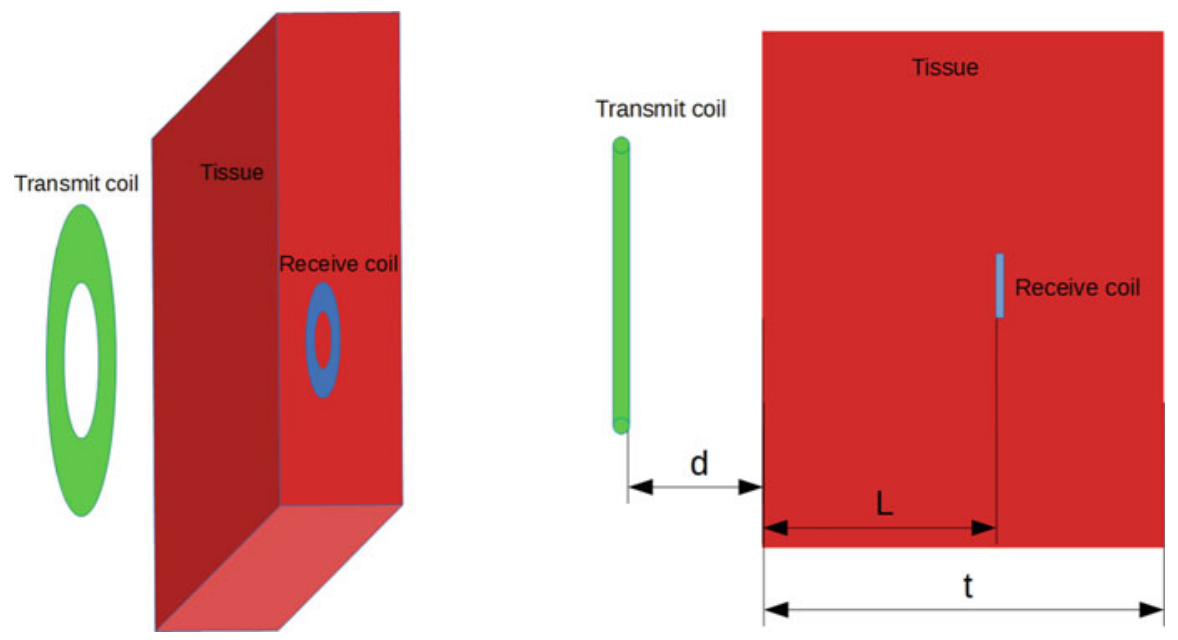

Fig. 1 Transmit coil, receive coil, and biological tissue 
field are only a tiny fraction of the power that is delivered to the implant. As a result, for various distances from the transmit coil, it is simply possible to calculate magnetic field of the transmit coil as though in air. However, the tissue losses, though small, are a crucial factor in exposure assessment.

To assess these losses, we look at the electric field generated by the time-varying magnetic field. The electric field leads to circulating eddies and causes heating. It is this heating that needs to be kept within limits, and the metric used in this regard is the specific absorption rate which is defined as the amount of heat energy absorbed in the tissue per $\mathrm{kg}$ and is expressed in $\mathrm{W} / \mathrm{kg}$.

To estimate SAR due to a certain transmit coil located near tissue, it is normally required to do full-wave simulations of the coil loaded with biological tissue using the software package such as Ansys HFSS. Furthermore, such simulations would need to be performed for a range of distances between the transmitter and the exposed tissue so that the safe standoff distance can be estimated. For each distance, one can calculate the maximum allowable current and input RF power that would correspond to maximum allowable SAR in the tissue. To do this work, it requires significant computational resources and time. Is there an easier way to estimate electric field and SAR in the tissue?

\section{Tissues}

There are four abundant tissues of the human body that are of particular interest for exposure assessment: skin, fat, muscle, and bone. Each one of these tissues exhibits different frequency-dependent material characteristics such as electric permittivity, conductivity, and mass density. Depending on the tissue properties at a given frequency of interest, we can identify the most lossy tissue type that will exhibit the highest SAR per unit field strength.

As an example, let us choose an ISM frequency, say $13.56 \mathrm{MHz}$. For the chosen frequency, the muscle tissue dissipates most power. For simulation purposes, we use a block of muscle tissue next to the transmit coil and study effect of the heating. The relative electric permittivity for the muscle tissue for the frequency that we chose is 138 [14] which indicates how much electric field is attenuated inside the body compared to the electric field in air. Additionally, conductivity of muscle tissue is $0.628 \mathrm{~S} / \mathrm{m}$. Conductivity also contributes to attenuation of the electric field inside the tissue. The following chapter discusses the electric field and its components.

\section{Two Components of Electric Field Inside the Tissue}

It is known from electromagnetics that electric field can be represented as a sum of two components: 


$$
\mathbf{E}=-\nabla \varphi-j \omega \mathbf{A}
$$

where $\varphi$ is the scalar electric potential (electric charge as a source) and $\mathbf{A}$ is the magnetic vector potential (electric current as a source). For our analysis, let us call - $\nabla \varphi$ as "charge" electric field and $-j \omega \mathbf{A}$ as "current" electric field. Then, the following holds good:

$$
\mathbf{E}=\mathbf{E}_{\text {charge }}+\mathbf{E}_{\text {current }}
$$

Transmit coil in a wireless charging system generates both types of electric field when powered. As the transmit coil approaches biological tissue, the "charge" and "current" components of electric field behave quite differently inside the tissue.

\section{1 “Charge" Electric Field}

Lines of "charge" electric field have beginning (positive charges) and end (negative charges) (Fig. 2).

As the electric charges accumulate on the transmit coil, the field lines originate and end on the coil (outside of the biological tissue). That is, every line of "charge" electric field enters the block of tissue and then exits it. This "charge" electric field is significantly attenuated (by a complex factor of $\varepsilon_{r}-j \frac{\sigma}{\omega \varepsilon_{0}}=138-j 833$ ) by charges that accumulate on surfaces of the block of tissue (Fig. 3).

Fig. 2 "Charge" component of electric field

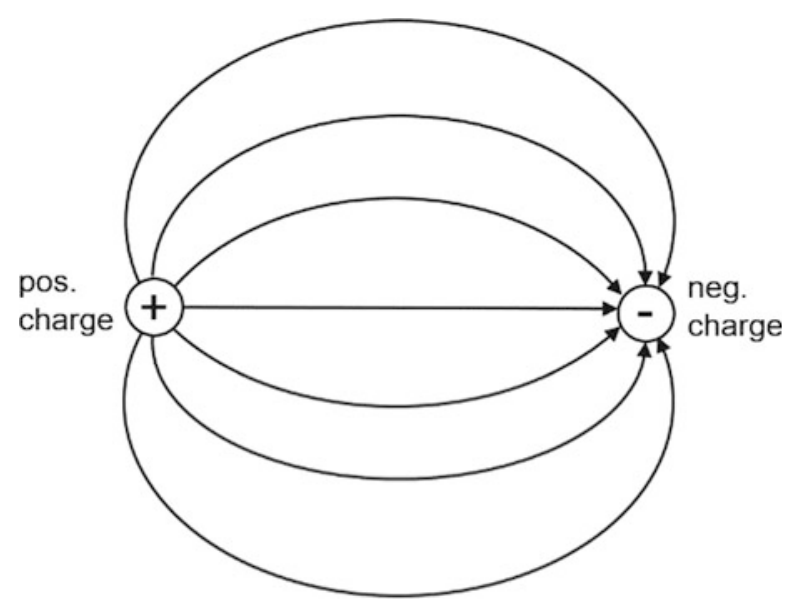


Fig. 3 Attenuation of "charge" electric field in the tissue

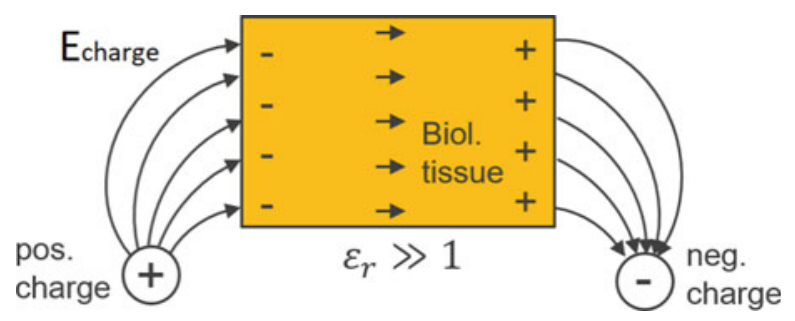

Fig. 4 Loop of current

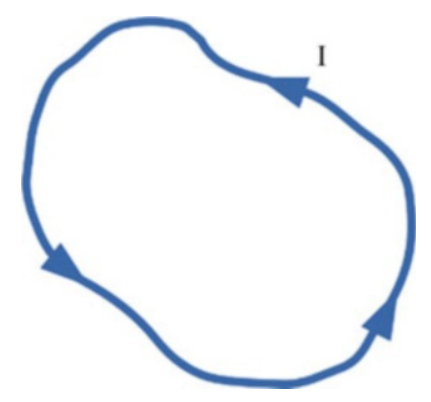

\section{2 “Current” Electric Field}

Generally speaking, the "current" electric field is not divergence-free, because the current in the coil is not the same at different points of the loop. However, if the selfresonance frequency of a transmit coil is high (compared to the chosen frequency), then the current can be considered to be the same throughout the coil. Let us consider a loop where the current $I$ is the same throughout as shown in the following figure (Fig. 4).

The "current" electric field of the loop can be written as follows:

$$
\mathbf{E}(\mathbf{r})=-\frac{j \omega \mu_{0}}{4 \pi} I \oint \frac{\exp \left(-j k\left|\mathbf{r}^{\prime}-\mathbf{r}\right|\right)}{\left|\mathbf{r}^{\prime}-\mathbf{r}\right|} d \vec{l}^{\prime}
$$

It can be shown that in free space, the divergence of "current" electric field of the loop of current is zero. This means that lines of this electric field have no beginning and no end, that is, they terminate into themselves. Just as magnetic field $\mathbf{B}$, the "current" electric field of the loop of current consists of self-terminating field lines (Fig. 5).

Some of these lines are located entirely inside the block of tissue. These lines are not attenuated by surface charges as these lines never cross the surface. If a round transmit coil is positioned parallel to the block of tissue, then the "current" component of the electric field is not attenuated inside the block of tissue. Hence, it is the "current" electric field that contributes to tissue heating (SAR). 
Fig. 5 Field lines of "current" electric field

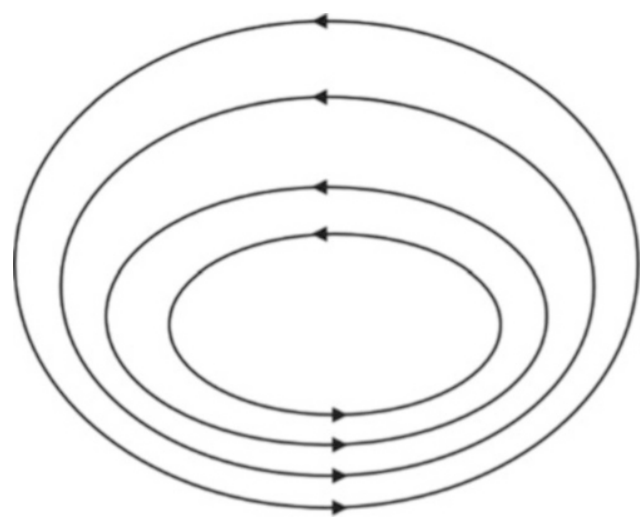

Table 1 Comparing loaded and unloaded simulations

\begin{tabular}{l|l|l|l|l}
\hline \multirow{2}{*}{$\begin{array}{l}\text { Distance, } \\
\mathrm{cm}\end{array}$} & \multicolumn{2}{|l|}{ HFSS, unloaded simulation } & \multicolumn{2}{l}{ HFSS, loaded simulation } \\
\cline { 2 - 5 } & Electric field of round coil & \multicolumn{2}{l}{ Electric field of round coil } \\
\cline { 2 - 5 } & Free space & $\begin{array}{l}\text { Value } \\
\mathrm{V} / \mathrm{m}\end{array}$ & $\begin{array}{l}\text { Value } \\
\mathrm{V} / \mathrm{m}\end{array}$ & Loaded with human tissue \\
\hline 10 & & 5.62 & 1.63 & \\
\hline
\end{tabular}

\subsection{Comparing Effects of Tissue Loading Using HFSS Simulations of Transmit Coil at $10 \mathrm{~cm}$ from Tissue}

When there is no tissue (unloaded), the electric field in the plane located $10 \mathrm{~cm}$ away from the coil is plotted. When tissue is present (loaded), the electric field on the surface of the $\varnothing 40 \mathrm{~cm}$-diameter and $6 \mathrm{~cm}$-tall cylinder of muscle tissue is plotted with the transmitting coil still $10 \mathrm{~cm}$ away.

From Table 1, we observe that the electric field generated by a transmit coil at a plane $10 \mathrm{~cm}$ from it is quite different if tissue is introduced. The presence of the tissue significantly attenuates overall electric field. Based on our previous definitions of types of electric fields, it is the "charge" electric field that is attenuated inside the tissue. 


\section{5 “Uniform Current" Approximation}

When an RF coil is excited, current develops in the coil and there is charge accumulation on different parts of the coil. Currents give rise to "current" electric field and charges produce "charge" electric field. As it is clear from the previous sections, it is current flowing in the RF coil that is responsible for "current" electric field that in turn contributes to SAR. If the coil is operating at the frequency that is several times lower than self-resonance frequency, it makes good sense to assume uniform current flowing throughout the RF coil. The coil can be modelled as a combination of short current elements (Fig. 6).

"Current" electric field from the short current element is as follows:

$$
\mathbf{E}=-\frac{j \omega \mu_{0}}{4 \pi} \cdot I \cdot \frac{\vec{l}}{r} \cdot \exp (-j k r)
$$

Each element contributes to the "current" electric field. Adding up the contributions of the elements, "current" electric field can be calculated and plotted, thereby providing an estimate for SAR. The following figure demonstrates how an RF coil can be formed from an arrangement of small current elements (Fig. 7).

There are several common coil geometries that we use in practice when performing the power transfer calculations. It is possible to write a code (MATLAB, $\mathrm{C}++$, or others) to generate several commonly used coil geometries from a combination of short wire elements (Fig. 8).

Given the input parameters (radius, pitch, etc.), most common coil geometries can be generated. Furthermore, for such coils, the values of magnetic field and "current" electric field can be calculated at any point in space.

We are interested in the effects of such coils on nearby human tissues (skin, muscle, fat, bone). As has been discussed, the "current" electric field may not

Fig. 6 Current interval and observation point

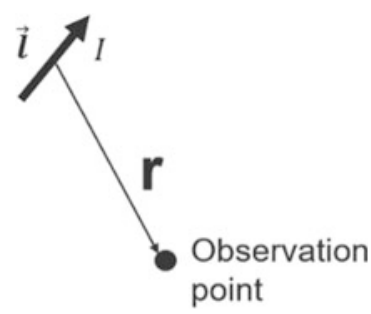

Fig. 7 Crude drawing of RF coil

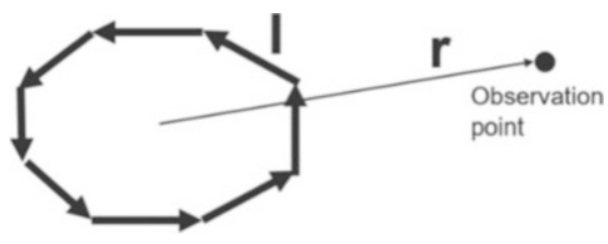


Fig. 8 Five common coils

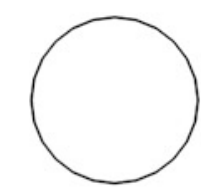

Round coil

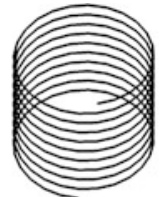

Solenoidal coil

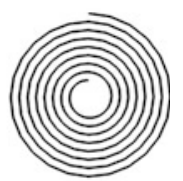

Spiral coil

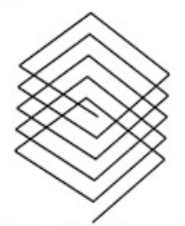

Square solenoidal coil

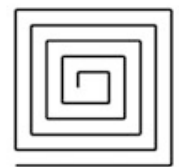

Square spiral coil

attenuate when it crosses the air-to-tissue boundary. The highest electric field (and SAR) develops on the surface of the body facing the transmit coil. We calculate the electric field on the surface of the body and find the maximum value of electric field (and SAR).

\section{Getting Rid of the Block of Tissue}

"Current" electric field is the main contributor to tissue heating (SAR) and it does not attenuate inside the tissue. This means that we can do an RF simulation of the transmit coil in the absence of the tissue using "uniform current" simulation and then use the result of this simulation ("current" electric field) to estimate maximum SAR (Fig. 9).

We calculate maximum value of SAR per unit input RF power at several planes located at various distances from the coil. We then scale the input RF power to bring SAR to the maximum permitted value. This way, for a range of distances from the coil, the maximum RF power for a given transmit coil can be obtained.

It is important to mention that HFSS simulations are expensive in terms of setup time, simulation time, data processing, and license cost. "Uniform current" simulations are cheap for each one of these terms.

\section{Maximum Allowed Current}

When moving the transmit coil away from the block of tissue, we can provide more RF power into the coil (so that the SAR limit is not exceeded) (Fig. 10).

The farther the tissue is from the coil, the larger the current it can take before hitting the SAR limits. 
Fig. 9 Replacing several full-wave loaded simulations by a single (not full-wave) unloaded "uniform current" simulation
Several

full-wave

loaded

simulations

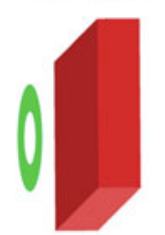

4

Single unloaded simulation

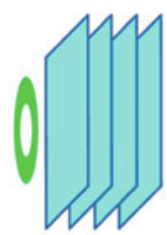

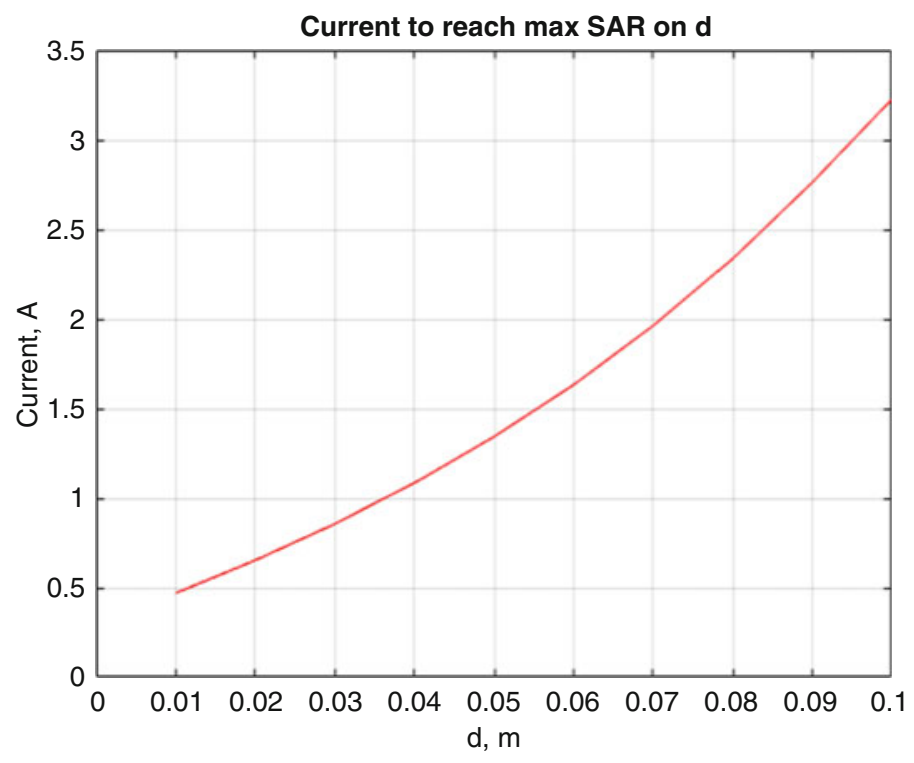

Fig. 10 Maximum allowed current on the distance to tissue, $I(d)$ 


\section{Verification: HFSS vs. "Uniform Current"}

To verify the idea that the electric field of a transmit coil inside the tissue is close to electric field in free space, two types of simulations are performed:

- HFSS full-wave simulations to plot electric field generated by a round transmit coil on the surface of the tissue ( $\varnothing 40 \mathrm{~cm}$ diameter and $6 \mathrm{~cm}$ tall) by positioning it at several distances from the coil.

- "Uniform current" simulation. We define a round coil carrying uniform current throughout and plot electric field on several planes parallel to the coil. This is not full-wave simulation.

We use one loop $\varnothing 17 \mathrm{~cm}$ round coil.

Maximum values of electric field differ by about $3 \%$.

Comparison between HFSS simulations and "uniform current" simulations is shown in Table 2.

Full-wave simulations done by HFSS are computationally intense and they require considerable time to perform. We see that the relatively simple "uniform current" simulation produces about the same result without using HFSS.

\section{Skin Depth Attenuation in the Tissue}

Above, we assumed that the presence of the tissue does not significantly change fields outside of the tissue. When can we make such an assumption? Our answer is that the skin depth should be significantly larger than the thickness of the tissue: $\delta \gg t$, which happens at low frequency. In the opposite limiting case (high frequency), skin depth is small, and there is a significant attenuation of external field right near the boundary. This would correspond to the case of metal (highly conductive material), where electric field is approaching zero. In case if the skin depth is comparable to the tissue thickness, we need a formula that would describe the attenuation of the external field near the boundary. The simplest way to describe the attenuation of the external field near the tissue can be done by introducing the exponent $\exp (-t / \delta)$. However, we get better agreement with full-wave simulations if we state that the attenuation is $\exp \left(-t^{\prime} / \delta\right)$, where $t^{\prime}$ is a weighted combination (inversely weighted) of thickness $t$, distance $d$, and radius (half size) of tissue block $R: t^{\prime}=1 /\left(\frac{1}{t}+\frac{1}{d}+\frac{1}{R}\right)$. Here is our rationale for doing such weighting:

- In case if $t \ll d$ and $t \ll R$, we have a straightforward exponential attenuation of field with depth $\exp (-t / \delta)$, that is, $t^{\prime} \approx t$. 
Table 2 Comparing loaded HFSS simulations and unloaded "uniform current" simulation

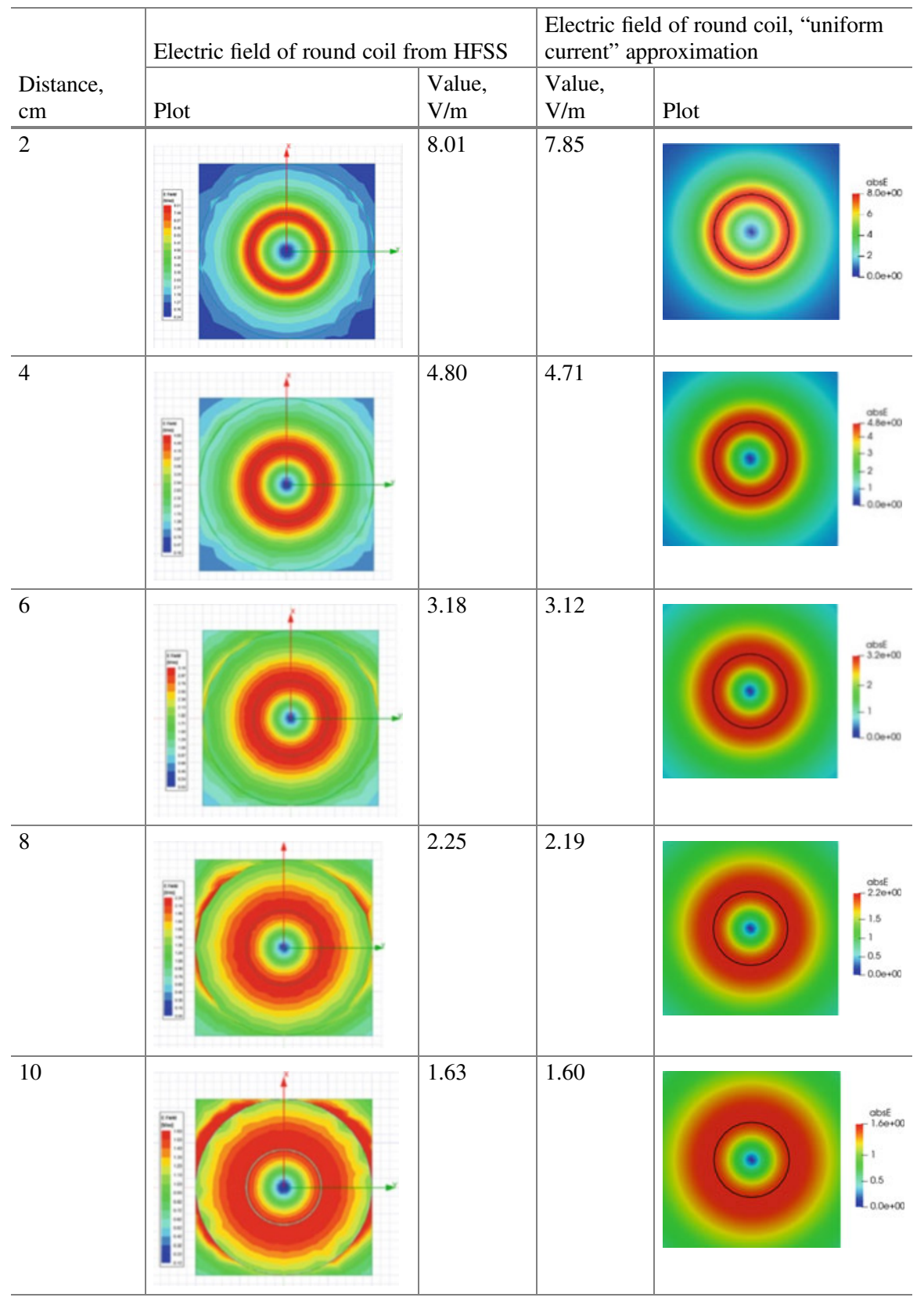




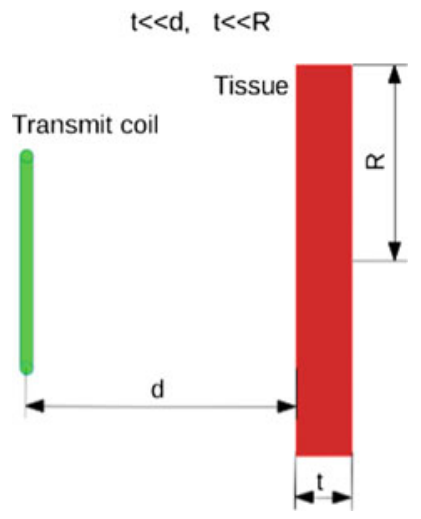

- In case if $d \ll t$ and $d \ll R$, the coil wiring is close to the tissue. Almost all induced current in the body is flowing right next to coil wiring. Effect of the tissue thickness $d$ and tissue size $2 R$ would be very small. So $t^{\prime} \approx d$.

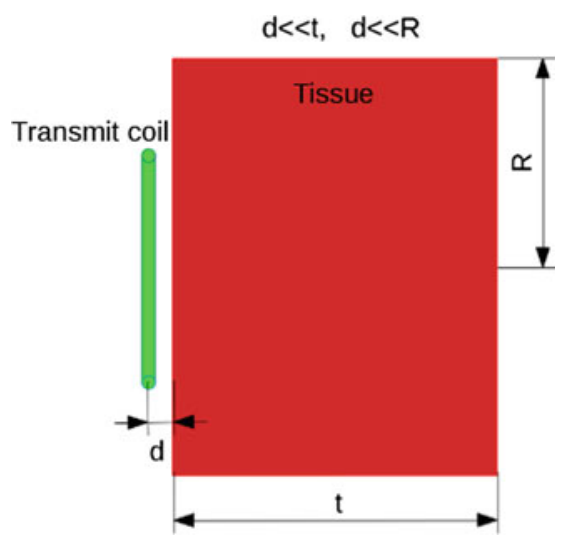

- In case if $R \ll r$ and $R \ll d$, the E-field infiltrates into the tissue from the sides. So $R$ plays a role of thickness: $t^{\prime} \approx R$. 


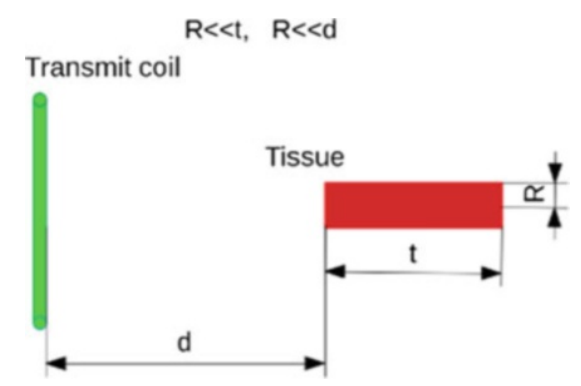

In the following figure, we plot dependence of the maximum electric field on conductivity (Fig. 11). We compare the "uniform current" approximation (theory) with HFSS simulations. In the "uniform current" approximation, we first calculate the maximum electric field and then attenuate it by a factor $\exp \left(-t^{\prime} / \delta\right)$. In HFSS simulation, we used a tissue disk having diameter of $2 R=20 \mathrm{~cm}$.

First of all, we see from the plot that increasing the value of $\varepsilon_{r}$ leads to the flatter HFSS curve on the left side of the graph (low conductivity). This is exactly in line with our claim that high $\varepsilon_{r}$ leads to significant attenuation of the "charge" electric field.

Secondly, for both HFSS and theoretical estimate ("uniform current" simulation), we observe a fall off at high conductivity $(\sigma>1 \mathrm{~S} / \mathrm{m})$. As a reference, the muscle conductivity at $13.56 \mathrm{MHz}$ is $0.628 \mathrm{~S} / \mathrm{m}$. To our opinion, there is a satisfactory agreement between HFSS simulations and the "uniform current" estimate.
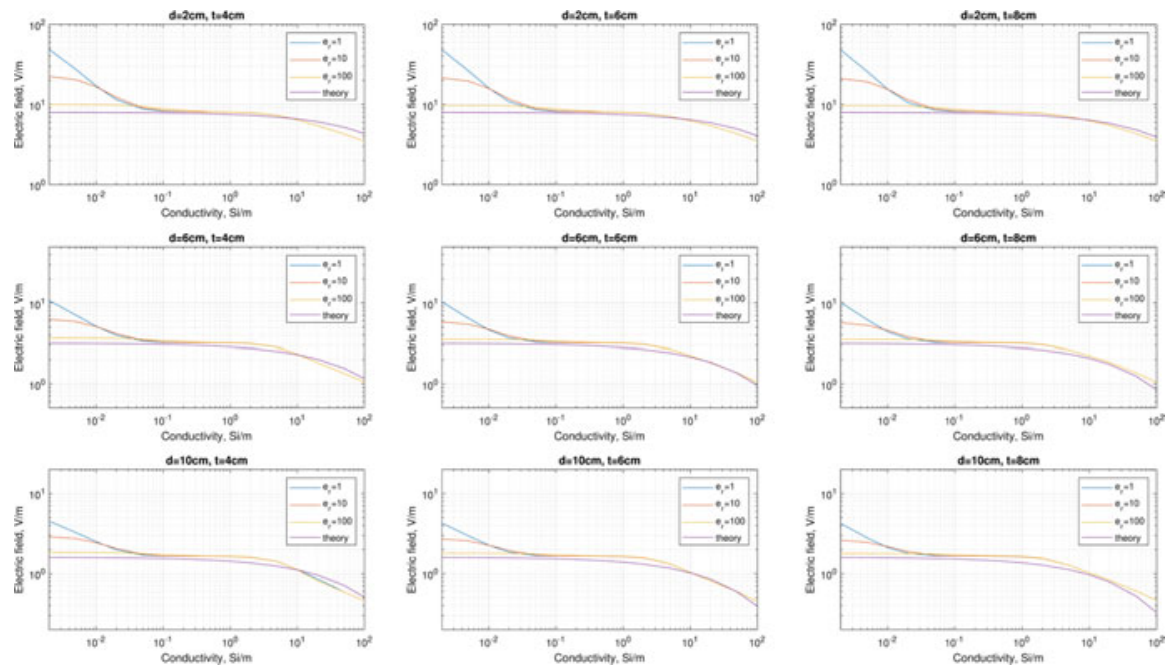

Fig. 11 Maximum electric field for a range of tissue conductivities $(0.002-100 \mathrm{~S} / \mathrm{m})$, three dielectric permittivities $(1,10,100)$, three coil-tissue distances $(2 \mathrm{~cm}, 6 \mathrm{~cm}, 10 \mathrm{~cm})$, and three tissue thicknesses $(4 \mathrm{~cm}, 6 \mathrm{~cm}, 8 \mathrm{~cm})$ 
"Uniform current" estimate works well to describe the maximum electric field (and therefore SAR) developing on the surface of the tissue near the RF coil.

Transition between the flat response on the left (low $\sigma$ ) and exponential decay on the right (high $\sigma$ ) occurs roughly when skin depth in tissue $\delta$ is comparable to $1 /\left(\frac{1}{t}+\frac{1}{d}+\frac{1}{R}\right)$.

\section{Conclusions}

We demonstrated that electric field inside the body can be satisfactorily estimated without full-wave simulation software using simple, time- and energy-efficient means.

Speaking about interaction of the RF coil with the human body, the following conclusions can be made:

- "Charge" component of electric field is significantly attenuated inside the biological tissue (if $\varepsilon_{r} \gg 1$ ) and generally causes negligible loss. "Current" component may not experience significant attenuation.

- It is the "current" component of electric field that is mostly responsible for heating the tissue.

- To estimate electric field and SAR inside the block of tissue located at certain distance, it may be sufficient to perform an unloaded simulation ("uniform current" simulation) that produces only "current" electric field.

\section{References}

1. Zeng, F. G., Rebscher, S., Harrison, W., Sun, X., \& Feng, H. (2008). Cochlear implants: System design, integration, and evaluation. IEEE Reviews in Biomedical Engineering, 1, 115-142.

2. Loeb, G. E., Peck, R. A., Moore, W. H., \& Hood, K. (2001). BionTM system for distributed neural prosthetic interfaces. Medical Engineering \& Physics, 23(1), 9-18.

3. Weiland, J. D., \& Humayun, M. S. (2014). Retinal prosthesis. IEEE Transactions on Biomedical Engineering, 61(5), 1412-1424.

4. Lemdiasov, R., \& Venkatasubramanian A. (2017). Transmit coil design for wireless power transfer for medical implants. In: 39th annual international conference of the IEEE engineering in medicine and biology society, July 11-15, 2017, Jeju Island, Korea.

5. Jegadeesan, R., \& Guo, Y.-X. (2012). Topology selection and efficiency improvement of inductive power links. IEEE Transactions on Antennas and Propagation, 60(10), 4846-4854.

6. RamRakhyani, A., \& Lazzi, G. (2013). On the design of efficient multicoil telemetry system for biomedical implants. IEEE Transactions of Biomedical Circuits and Systems, 7(1), 11-23.

7. Jegadeesan, R., Nag, S., Agarwal, K., Thakor, N., \& Guo, Y.-X. (2015). Enabling wireless powering and telemetry for peripheral nerve implants. IEEE Journal of Biomedical and Health Informatics, 19, 958-970.

8. Christ, A., Douglas, M. G., Roman, J. M., Cooper, E. B., Sample, A. P., Waters, B. H., Smith, J. R., \& Kuster, N. (2013). Evaluation of wireless resonant power transfer systems with human 
electromagnetic exposure limits. IEEE Transactions on Electromagnetic Compatibility, 55(2), 265-274.

9. Makarov, S., Horner, M., \& Noetscher, G. (2018). Brain and human body modeling. Cham: Springer.

10. Jackson, J. D. (1962). Classical electrodynamics. New York: John Wiley \& Sons.

11. Stratton, J. A. (1941). Electromagnetic theory. New York: John Wiley \& Sons.

12. "http://niremf.ifac.cnr.it/tissprop". Accessed 6 Jan 62020.

13. 47 CFR 1.131, "https://www.fcc.gov/general/fcc-policy-human-exposure". Accessed 6 Jan 2020.

14. ICNIRP guidelines for limiting exposure to time-varying electric, magnetic and electromagnetic fields (up to $300 \mathrm{GHz}$ ), "https://www.icnirp.org/cms/upload/publications/ICNIRPemfgdl.pdf". Accessed 6 Jan 2020.

Open Access This chapter is licensed under the terms of the Creative Commons Attribution 4.0 International License (http://creativecommons.org/licenses/by/4.0/), which permits use, sharing, adaptation, distribution and reproduction in any medium or format, as long as you give appropriate credit to the original author(s) and the source, provide a link to the Creative Commons license and indicate if changes were made.

The images or other third party material in this chapter are included in the chapter's Creative Commons license, unless indicated otherwise in a credit line to the material. If material is not included in the chapter's Creative Commons license and your intended use is not permitted by statutory regulation or exceeds the permitted use, you will need to obtain permission directly from the copyright holder. 